\title{
How to Survive Flying Too Close to the Sun
}

\author{
The Parker Solar Probe has flown through the Sun's atmosphere-an \\ unforgiving environment that poses a number of engineering challenges.
}

\author{
By Michael Schirber
}

n spring of this year, the Parker Solar Probe got closer to our star than any previous spacecraft, dipping, for the first time, into a part of the Sun's corona that is "magnetically dominated" (see Viewpoint: Momentous Crossing of a Solar Boundary and To Touch the Sun). The probe's instruments recorded fluctuations in the magnetic field and in the plasma energy-information that will help theorists better understand the heating of the corona and the origin of the solar wind. As the mission continues, the probe will fly even closer, with its final orbit in 2025 bringing it within 9 solar radii (about 3.83 million miles) of the Sun's surface.

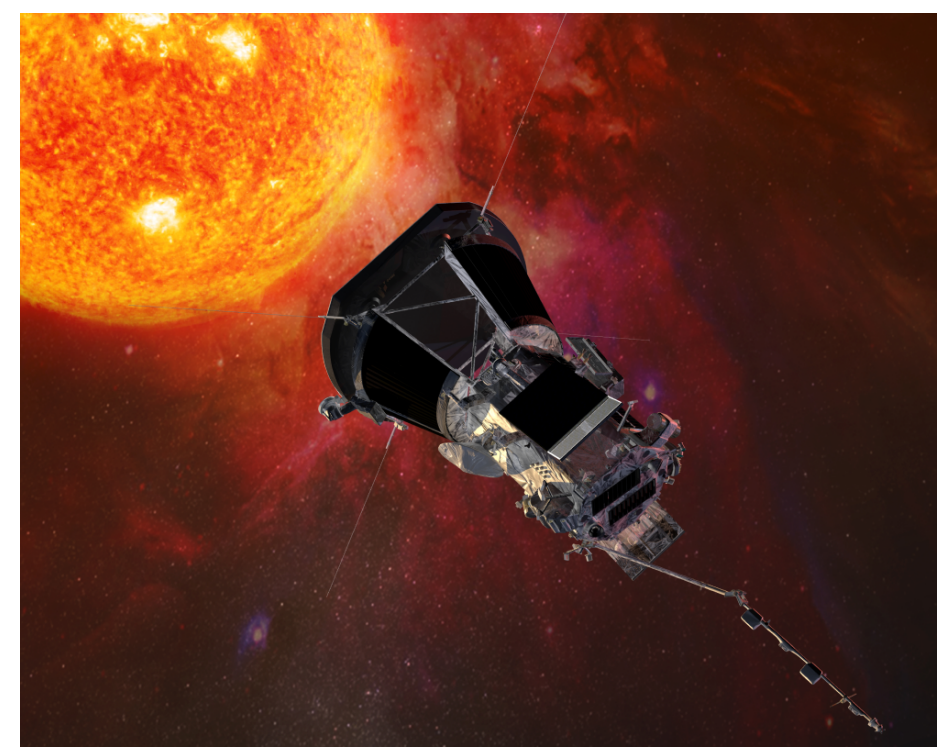

An artist's illustration of the Parker Solar Probe getting up close and personal with the Sun.

Credit: Johns Hopkins University Applied Physics Laboratory
One may wonder what dangers await a spacecraft that goes up to a star's front door. "There are many ways in which even a normal spacecraft can fail, but all of those risks become greater close to the Sun," says Tony Case of the Harvard-Smithsonian Center for Astrophysics in Massachusetts, a member of the probe's Solar Wind Electrons Alphas and Protons (SWEAP) team. Here is a rundown of the main challenges that the Parker Solar Probe engineers had to tackle in sending their spacecraft where Greek heroes failed to reach.

\section{Beating the Heat}

The Sun is about 475 times brighter at the probe's closest approach than it is at Earth's orbit. That translates into 5.5 MW of sunlight pounding on the spacecraft's front surface. The probe is designed to reflect much of the incoming light away, but even so, exposed surfaces will get as hot as $1500^{\circ} \mathrm{C}$. (The plasma in the corona is around one million degrees, but it is too sparse to transfer much heat to the probe.)

Normal materials wouldn't survive at the maximum temperatures. Many metals melt or turn soft; epoxies evaporate into the vacuum; and most insulators stop insulating. "There are very few elements on the periodic table that function at those temperatures," says Justin Kasper from BWX Technology and the University of Michigan, who leads the SWEAP team.

Some metals, such as tungsten, have high melting temperatures, but they would make a lousy thermal shield. "These metals are heavy and actually conduct heat pretty well," says Betsy Congdon from Johns Hopkins University in Maryland, who is the lead engineer for the probe's Thermal Protection System. A heavy shield would require more powerful 


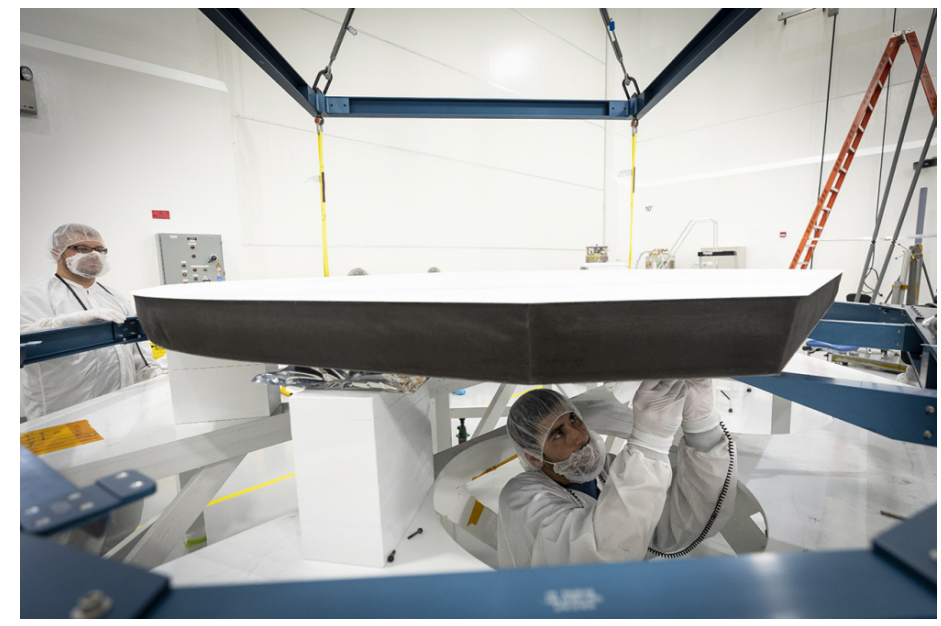

The probe's 2.4-m-wide heat shield was designed to be not only durable but also lightweight, with a mass of only $72 \mathrm{~kg}$.

Credit: NASA/Johns Hopkins APL/Ed Whitman

(more expensive) rockets. A highly conductive shield would allow too much heat to flow back to the instruments.

The probe's actual shield is made of carbon in a 11.5-cm-thick sandwich structure that only weighs $72 \mathrm{~kg}$. "The top and bottom sheets are made of carbon-carbon, which is a high-temperature version of composites like you find in golf clubs or tennis rackets," Congdon says. In between these sheets is a carbon foam whose volume is $97 \%$ empty space. This porous structure reduces heat conduction.

To reflect away as much light as possible, the front surface of the shield has a thin coating of white ceramic paint that was developed particularly for the probe. Engineers carefully sprayed on this paint to eliminate any bumps that could produce uneven heating on the surface.

Behind the shield, the temperatures rapidly drop, with most of the instruments experiencing room-like temperatures of around $30^{\circ} \mathrm{C}$. The large temperature gradient between the front and back of the probe can lead to high mechanical stresses. "You wind up creating huge forces that can rip the thing apart," Kasper says. Engineers installed flexible joints that allow the probe to bend in respond to these thermal forces.

\section{Catching the Wind}

Most of the probe's instruments stay tucked behind the shield,

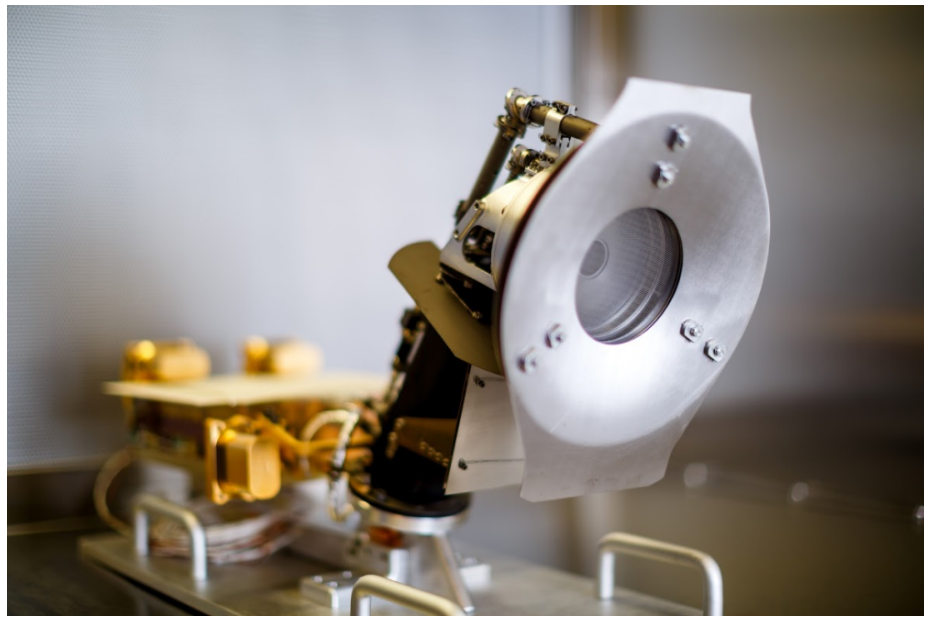

The Solar Probe Cup (SPC) sits outside of the shadow of the probe's main heat shield. It has its own heat shield (shown here) made of niobium alloy. The central aperture holds the metal grid and the metal plate sensor.

Credit: A. Wang/andrewtakesphotos.com

but the Solar Probe Cup (SPC) sticks out in the blazing sunlight, measuring the charged particles of the solar wind. "We have this unique problem with the SPC of trying to allow in the particles we want to measure, while also dealing with all the light and heat that comes with them," Case explains.

To make the SPC robust, Case and Kasper and their colleagues designed it to be bare-bones. The front of the cup is a metal grid that produces an electric field for controlling which charged particles get through to the sensor. In a lab environment, that particle sensor might be a CCD camera or a microchannel plate, but such a sensitive device would be overwhelmed by heat and photons from the Sun. Instead, the SPC team used a no-frills metal plate that generates a picoamp current from the solar wind particles that smack into it.

Although the design is simple, finding the right materials for the SPC was not. The team tested samples using a large mirror array that focuses megawatts of sunlight onto a target position. The researchers ran similar tests in a lab using four IMAX film projectors to simulate the Sun. They found that only a few types of elements could take such a beating. "Niobium, tungsten, molybdenum. That's pretty much it for the main constituents of metals that we could use in the SPC instrument," says Case. 


\section{RESEARCH NEWS}

Besides metals, the SPC designers needed heat-resistant electrical insulators. The list of candidates was small, and only sapphire made the grade. The team obtained single-crystal sapphire pieces and placed them around the niobium wires that carry $8000 \mathrm{~V}$ to the cup's electric-field grid.

\section{Going Alone}

The intense sunlight does more than roast the probe. It also exerts a strong radiation pressure that pushes on the shield and could flip the spacecraft around in about $30 \mathrm{~s}-$ if the spacecraft wasn't actively correcting its position, Kasper says. That sort of flipping would be disastrous, as it would quickly expose the components in the back of the probe and cause them to fry.

The brightness of the Sun also wreaks havoc with communication. The Sun is bright at many wavelengths, including the microwave channel that NASA uses to exchange messages with the spacecraft. "It can be difficult to 'hear' the spacecraft over the signal from the Sun," Kasper says. Thus there are weeklong periods where the probe is out of total communication with Earth. "We just have to wait and hope the spacecraft takes care of itself," Kasper says.

To deal with the communication gaps, the spacecraft was programmed to be highly autonomous. Sensors on its side detect how much sunlight is peeking around the shield. If that light becomes too bright, reaction wheels on the probe automatically change their spin rate, causing an opposite rotation in the spacecraft that keeps it pointing at the Sun. When the probe reaches a safe distance from the Sun, it can "relax" by deploying its solar panels (to recharge) and its high-gain antenna (to exchange data with Earth).

\section{Dealing with Speed}

Just getting to the Sun was difficult. Earth moves around the Sun at $30 \mathrm{~km} / \mathrm{s}(67,000 \mathrm{mph})$, so a spacecraft needs to lose much of that angular momentum in order to drop down into a
"low-Sun orbit." The probe was launched in August 2018 on a Delta IV Heavy rocket-one of the largest launch vehicles around. Inside that rocket, the probe had its own rocket to slow it down in space. "And even that wasn't enough to get us into our close approach-we have to 'cheat' by using a whole bunch of gravity assists with Venus," Kasper says. The probe has passed near Venus five times and will do so two more times before the end of the mission. Each flyby transfers orbital energy from the spacecraft to the planet.

But all that slowing down doesn't mean the probe is dawdling along. The spacecraft has an elliptical orbit that swings it by the Sun at about $200 \mathrm{~km} / \mathrm{s}(400,000 \mathrm{mph})$-the fastest that any human-made object has ever gone. That speed poses a danger, as the probe could run into interplanetary dust particles. Such a collision could blow a coin-size hole through a metal sheet, Kasper warns. For that reason, crucial components on the probe were surrounded by dense materials.

Fast-moving particles could also cause damage to the spacecraft. Every so often, the Sun erupts in a solar flare or a coronal mass ejection. "The radiation goes up by orders of magnitude when one of these big events happens," Case says. One concern is that this extra radiation will affect computer data. To mitigate this danger, engineers installed three separate copies of vital information in the onboard memory. "Every time the probe uses that memory, it looks at all three copies," Case explains. A kind of vote is taken, where any data corruption is assumed to be the minority. "If there's a solar flare, we can deal with that penetrating radiation, and it doesn't cause any significant effects within the spacecraft."

Michael Schirber is a Corresponding Editor for Physics based in Lyon, France. 